\title{
|||||||||||||||||||||||||||||||||||||||||||||||||||||||||||||||||||.
}

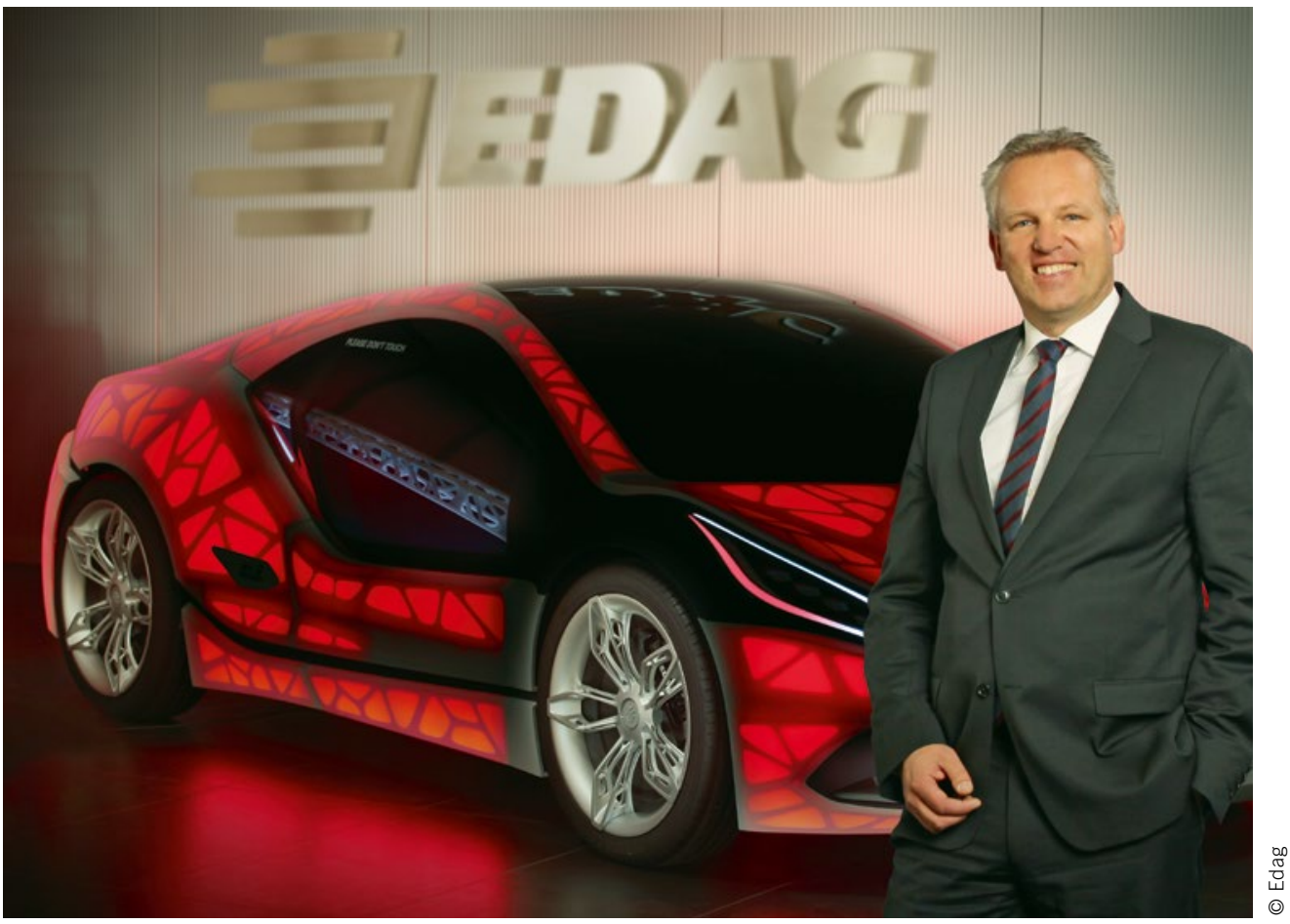

Dipl.-Ing. Jörg Ohlsen

CEO der Edag Engineering $\mathrm{GmbH}$

\section{Beherrschung der Variantenvielfalt}

Der Trend ist ungebrochen: In den vergangenen Jahren kam es zu immer mehr Fahrzeugmodellen in der Automobilindustrie. Das Nachfrageverhalten der Kunden sowie die zunehmende Internationalisierung der Absatzmärkte und die damit notwendigen Lokalisierungen haben diese Entwicklung weiter verstärkt. Parallel sehen sich die Entwickler zudem mit der Integration von alternativen Antriebskonzepten und Energiespeichersystemen konfrontiert. Die resultierenden Packagesituationen wirken sich verschieden auf die Auslegung der Karosserie und die Gewichtsverteilung im Gesamtfahrzeug aus. Die Vielfalt an Anpassungen ist somit vorprogrammiert.

In der Konsequenz sind die Herausforderungen an eine flexible Gestaltung der Karosseriekonzepte und der Produktionsverfahren exponentiell gestiegen - insbesondere, wenn diese Konzepte zunächst in sehr kleinen Stückzahlszenarien umzusetzen sind. Selbstverständlich sind wir weiterhin gefordert, das Thema Leichtbau voranzutreiben, um einen Beitrag für nachhaltige Produkte zu erbringen und gleichzeitig vertretbare wirtschaftliche Lösungen zu erarbeiten.

Der Spagat zwischen Varianz, Stückzahlszenarien, Kosten und Leichtbau ist mit aktuellen Strategien immer schwieriger zu bewältigen. Alle Engineeringunternehmen sind gefordert, querzudenken und alternative Karosserie- und Fertigungskonzepte zu identifizieren. In der Kombination aus generativ gefertigten Bauteilen mit bionisch inspiriertem Design sehen wir einen aussichtsreichen Weg. Mit dem Konzeptfahrzeug „Edag Light Cocoon“ haben wir die Vision einer werkzeugarmen Fertigung einer Karosserie bei gleichzeitig ultraleichten Strukturen bereits anmoderiert.

Eine solch umfängliche generative Fertigung ist zwar heute noch nicht serienreif umsetzbar; partielle Lösungen sehen wir jedoch als mittelfristig machbar an. So haben wir mit dem Laser-Zentrum Nord, Concept Laser und der BLM Group ein bionisch optimiertes, hybrid gefertigtes Spaceframekonzept für einen Karosserieknoten entwickelt. Sein Erfolg liegt in der Kombination von generativ hergestellten Karosserieteilen und konventionell gefertigten Profilen. Die Stahlknoten können dank generativer Fertigung hochflexibel und multifunktional gestaltet werden, um beispielsweise unterschiedliche Fahrzeugvarianten ohne zusätzliche Werkzeuge, Betriebsmittel und Anlaufkosten in einer hochflexiblen Produktionszelle „on demand“ produzieren zu können. Das Ergebnis zeigt einen bionisch gestalteten und lastpfadoptimierten Spaceframeknoten.

Durch den Einsatz vorrichtungs- und werkzeugarmer Verfahren können zukünftig alle Karosserievarianten wirtschaftlich und mit höchstmöglicher Flexibilität gefertigt werden. Wir werden die Potenziale der generativen Fertigung sukzessive in konkrete Anwendungsfälle überführen, um eine zukunftssichere Antwort zur Beherrschung der Variantenvielfalt $\mathrm{zu}$ geben. 